\title{
Achievable rates for full-duplex massive MIMO systems with low-resolution ADCs/DACs under imperfect CSI environment
}

\author{
Juan Liu ${ }^{1 \dagger}$, Jianxin Dai ${ }^{2+}$ (D), Jiangzhou Wang ${ }^{3}$, Xiaohui Yinn², Zhifang Jiang ${ }^{2}$ and Jinyuan Wang ${ }^{1}$
}

\begin{abstract}
We investigate the uplink and downlink achievable rates of full-duplex (FD) massive multi-input multi-output (MIMO) systems with low-resolution analog-digital converters/digital-to-analog converters (ADCs/DACs), where maximum ratio combining/maximum ratio transmission (MRC/MRT) processing are adopted and imperfect channel state information (CSI) is assumed. In this paper, the quantization noise is encapsulated as an additive quantization noise model (AQNM). Then, employing the minimum mean-square error (MMSE) channel estimator, approximate expressions of the uplink and downlink achievable rates are derived, based on the analysis of the quantization error, loop interference $(\mathrm{LI})$, and the inter-user interference (IUI). It is shown that the interference and noise can be eliminated by applying power scaling law properly and increasing the number of antennas. Moreover, given the number of antennas, it is found that the uplink and downlink approximate achievable rates will converge to a constant when the number of quantization bit tends to infinity. Therefore, the system performance that can be improved by increasing ADC/DAC resolution is limited, implying that it is reasonable to adopt low-resolution ADCs/DACs in FD massive MIMO systems.
\end{abstract}

Keywords: Full-duplex, Massive MIMO, Low-resolution ADCs/DACs, Imperfect CSI, Achievable rates

\section{Introduction}

Equipped with a large number of antennas, massive MIMO system can effectively exploit the space resources to enhance the system spectral efficiency. On the other hand, full-duplex (FD) technology can double spectral efficiency theoretically since it simultaneously transmits and receives signals on the same frequency resources and enables two-way communication. However, how to eliminate the loop interference (LI) caused by FD is one of the major problems. In order to reap the benefits of these two key techniques, many papers have studied one massive MIMO system that is endowed with the FD functionality [1-5].

However, the base station (BS) with a large number of antennas will incur excessive hardware cost and radio

\footnotetext{
*Correspondence: daijx@njupt.edu.cn

†Juan Liu and Jianxin Dai contributed equally to this work.

${ }^{2}$ School of Science, Nanjing University of Posts and Telecommunications, Wenyuan Road, 210023 Nanjing, China

Full list of author information is available at the end of the article
}

frequency (RF) circuit power consumption. In particular, each receive antenna needs to be equipped with one analog-digital converter (ADC) unit, and each transmit antenna needs to be equipped with one digital-to-analog converter (DAC) unit. A typical flash ADC with $b$-bit and the sampling frequency $f_{s}$ operates $f_{s} 2^{b}$ conversion steps per second [6]. Therefore, using high-resolution ADCs can result in excessive overhead and power consumption, which is a major bottleneck in implementing massive MIMO systems. One way to deal with this issue is to adopt low-resolution ADCs [7]. Recently, many papers have studied the impact of low-resolution ADCs on massive MIMO systems. In [8], massive MIMO systems with low-resolution ADCs was studied and the uplink achievable rate was derived under Rayleigh fading channel. Then, this work was extended to the Rician fading channel in [9], where the uplink spectral efficiency (SE) was derived. Jia et al. [10] considered a more general FD massive MIMO relaying system where the relay is equipped with low-resolution ADCs, and the authors derived the approximated expression of the sum rate. 
Jiao et al. [11] assume that the receive antennas of the FD mode relay are equipped with low-resolution ADCs and [12] consider that low-resolution ADCs are used at both the relay and users. Kong et al. [13] studied the transmit processing with low-resolution DACs based on a minimum mean-square error (MMSE) approach. However, the low-resolution ADCs are not used at the transmit antennas of the relay in the above literature.

The main contributions of our works are as follows:

- We consider a FD massive MIMO system where the receive and transmit antennas are equipped with low-resolution ADCs/DACs is proposed in this paper. With the aid of additive quantization noise model (AQNM) and MMSE criterion, the uplink and downlink asymptotic achievable rates under imperfect channel state information (CSI) environment are derived.

- However, when the low-resolution ADCs/DACs are used at the receive and transmit antennas of the FD mode BS simultaneously, it is urgent to suppress the influence of the LI and study the effect of the quantization error on the system performance. In order to analyze the uplink and downlink asymptotic achievable rates intuitively, we study the effect of the quantization error, LI, and the inter-user interference (IUI). The following three cases are considered as follows: (1) with fixed $P_{U}, P_{D}$, and $M$, when $b \rightarrow \infty$ $\left(\alpha_{u}=\alpha_{d}=1\right)$; (2) with fixed $b$ and $M$, when $P_{U}=P_{D} \rightarrow \infty$; (3) assuming that the transmission power of BS and each user are scaled with $M$, i.e., $P_{U}=E_{U} / M$ and $P_{D}=E_{D} / M$, where $E_{U}$ and $E_{D}$ are fixed values.

- Based on the derived asymptotic expressions of the uplink and downlink achievable rates, we show that the multi-user interference, LI, quantization noise, and additive white Gaussian noise (AWGN) can be eliminated by applying power scaling law properly, increasing the number of antennas and ADC/DAC resolution. However, the system performance that can be improved by increasing ADC/DAC resolution is limited, implying that it is reasonable to adopt low-resolution ADCs/DACs in FD massive MIMO systems.

This paper is organized as follows. Section 2 introduces the FD massive MIMO system model, where the receive and transmit antennas are equipped with low-resolution ADCs/DACs. We derive a approximate expression of the uplink and downlink achievable rates for imperfect CSI in Section 3. Then, we analyze the impact of several interferences/noise including multi-user interference (MUI), LI, and noise on achievable rates. The numerical simulation results is analyzed in Section 4. Conclusions are drawn in Section 5.
Notations: The symbols used in this paper are as follows: $(A)^{T},(A)^{H}, \operatorname{tr}(A),\|A\|, \mathbb{E}\{\bullet\}$ represent the matrix transpose, conjugate transpose, matrix trace, the Euclidean norm, and expectation, respectively; $[\mathbf{A}]_{n n}$ represents the $n \times n$ diagonal entry of matrix $\mathbf{A} ; \mathbf{I}_{M}$ denotes $M \times M$ identity matrix; and $x_{i} \sim \mathcal{C N}\left(0, \sigma_{i}^{2}\right)$ denotes the $i$ th entry of a circularly symmetric complex-Gaussian vector $\mathbf{x}$, whose mean and variance are 0 and $\sigma_{i}^{2}$, respectively.

\section{System model}

As shown in Fig. 1, we study a single-cell FD massive MIMO system, which has $N$ users, assuming that BS is equipped with $2 M$ antennas ( $M$ antennas for transmission and $M$ antennas for reception) and each user has two antennas (one for transmission and one for reception) [14]. The downlink and uplink channel matrices are denoted as $\mathbf{G}_{D}=\left[\mathbf{g}_{D, 1}, \cdots, \mathbf{g}_{D, N}\right] \in \mathbb{C}^{M \times N}$ and $\mathbf{G}_{U}=$ $\left[\mathbf{g}_{U, 1}, \cdots, \mathbf{g}_{U, N}\right] \in \mathbb{C}^{M \times N}$, respectively. For simplicity, we decompose the downlink and uplink channel matrices as $\mathbf{G}_{a}=\mathbf{H}_{a} \mathbf{D}_{a}^{1 / 2}(a \in\{D, U\})$, where $\mathbf{H}_{a} \in \mathbb{C}^{M \times N}$ represents the $M \times N$ fast fading channel matrix, which has independent and identically distributed (i.i.d.) elements, each of which follows the distribution of $\mathcal{C N}(0,1), \mathbf{D}_{a}$ is the $N \times N$ diagonal matrix and its $n$th diagonal entry is $\left[\mathbf{D}_{a}\right]_{n n}=\beta_{a, n}$, which represents the large-scale channel fading. The LI channel matrix between transmit antennas and receive antennas of the BS are denoted as $\mathbf{G}_{L I} \in$ $\mathbb{C}^{M \times M}$, whose entries are i.i.d. and follow the distribution of $\mathcal{C N}\left(0, \mu_{L I}^{2}\right)$. Let us denote $\mathbf{G}_{I U}=\left[g_{I U, 1}, \cdots, g_{I U, N}\right] \in$ $\mathbb{C}^{N \times 1}$ as IUI channel with $\left[\mathbf{G}_{I U}\right]_{i, j}$ representing the channel coefficient from the $i$ th user to the $j$ th user. All the elements in $\mathbf{G}_{I U}$ are assumed to be i.i.d. with the distribution of $\mathcal{C N}\left(0, \delta_{i j}^{2}\right)$. Moreover, $\left[\mathbf{G}_{I U}\right]_{n n}=g_{n n}$ denotes the coefficient of self-interference channel of the $n$th user. To reduce the hardware cost and the power consumption, it is assumed that the receive antennas and transmit antennas at the BS are both equipped with low-resolution ADCs/DACs as shown in Fig. 1.

It is difficult to obtain the perfect CSI in the practical massive MIMO system. Therefore, the imperfect CSI is assumed in this paper. We consider a transmission within the coherence interval $T$ and use $\tau$ symbols for pilots. The power of pilot symbols is $P_{p}$, while the MMSE estimate of $\mathbf{G}_{a}$ is $\hat{\mathbf{G}}_{a}$. The channel estimation error matrix which is independent of $\mathbf{G}_{a}$ can be expressed as

$$
\Delta \mathbf{G}_{a} \triangleq \mathbf{G}_{a}-\hat{\mathbf{G}}_{a} .
$$

The variance of element of $\Delta \mathbf{G}_{a}$ are defined as $\Delta o_{a, n}^{2}=$ $\frac{\beta_{a, n}}{1+\tau P_{p} \beta_{a, n}}[13]$.

The uplink received signal $\mathbf{y}_{U}$ at the BS before the ADCs and the downlink un-quantized transmit signal $\mathbf{y}_{D}$ can be expressed as 


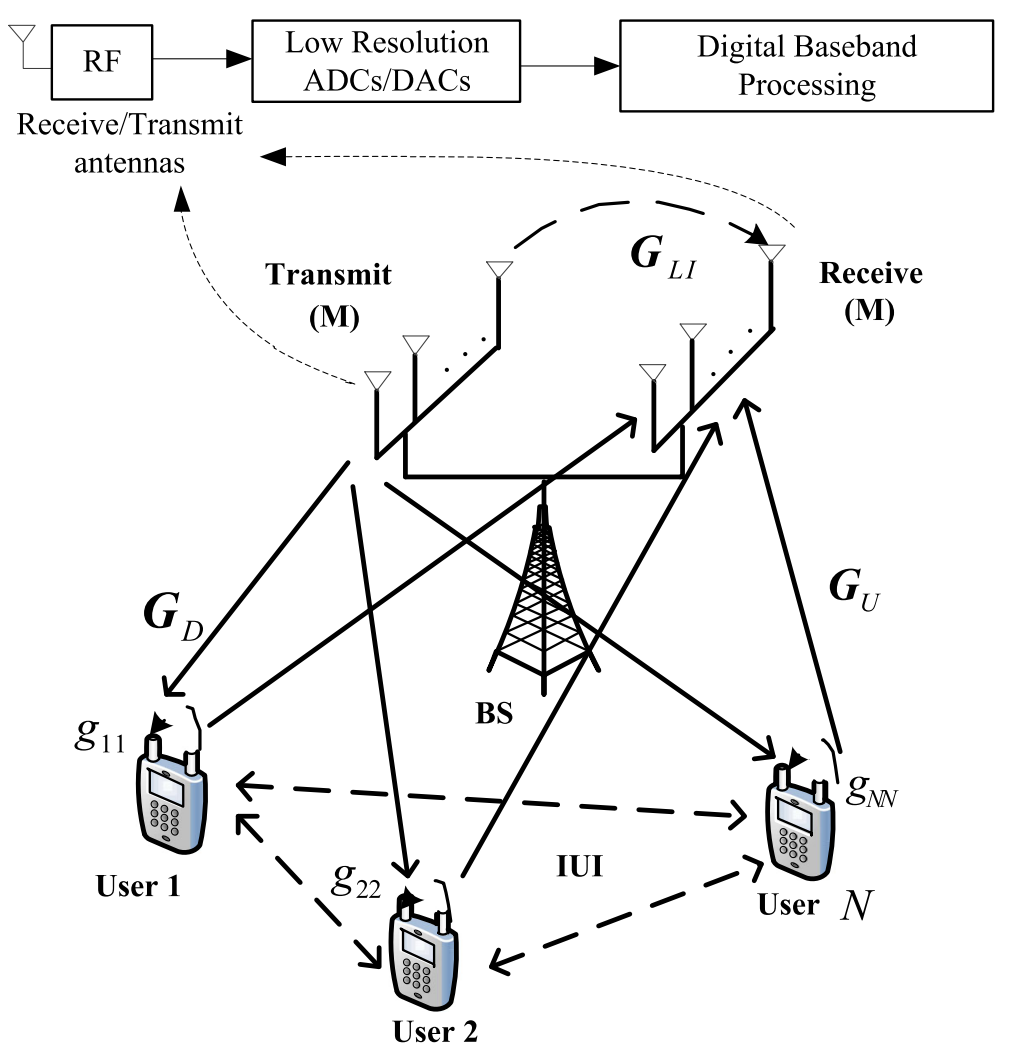

Fig. 1 System model

$$
\begin{gathered}
\mathbf{y}_{U}=\sqrt{P_{U}} \mathbf{G}_{U} \mathbf{x}_{U}+\sqrt{\eta} \sqrt{P_{D}} \mathbf{G}_{L I} \tilde{\mathbf{y}}_{D}+\mathbf{n}_{U}, \\
\mathbf{y}_{D}=\mathbf{W} \mathbf{x}_{D},
\end{gathered}
$$

where $\tilde{\mathbf{y}}_{D}$ is the quantized signal of the transmit antennas at the BS, and $P_{U}$ and $P_{D}$ are the transmission power of the users and $\mathrm{BS}$, respectively. $\mathbf{W}=\left[\mathbf{w}_{1}, \ldots, \mathbf{w}_{N}\right] \in$ $\mathbb{C}^{M \times N}$ represents the downlink precoding matrix. And $\mathbf{x}_{D}=\left[x_{D, 1}, \cdots, x_{D, N}\right]^{T}, \mathbf{x}_{U}=\left[x_{U, 1}, \cdots, x_{U, N}\right]^{T}$ are the transmit signal vectors of the BS and users, respectively, satisfying $\mathbb{E}\left\{\mathbf{x}_{a} \mathbf{x}_{a}^{H}\right\}=\mathbf{I}_{N}(a \in\{D, U\}) . \boldsymbol{n}_{U} \sim\left(0, \sigma_{U}^{2} \boldsymbol{I}_{M}\right)$ denotes the AWGN vector at the BS. $\eta$ is the factor that depends upon the extent to which the LI is eliminated.

In this paper, we adopt the AQNM which has been applied widely, and the uplink received signal at the BS $\mathbf{y}_{U}$ and the downlink transmit signal $\mathbf{y}_{D}$ after the ADCs/DACs can be processed as

$$
\begin{gathered}
\tilde{\mathbf{y}}_{U}=\alpha_{u} \mathbf{y}_{U}+\mathbf{q}_{U}, \\
\tilde{\mathbf{y}}_{D}=\alpha_{d} \mathbf{y}_{D}+\mathbf{q}_{D},
\end{gathered}
$$

with $\alpha_{u}=1-\rho_{u}$ and $\alpha_{d}=1-\rho_{d}$, where $\rho_{u}$ and $\rho_{d}$ are the inverse of the signal-to-noise and quantization noise ratios for the uplink and downlink, respectively, which depend on the number of quantization bits. For the non-uniform MMSE quantizer, the values of $\rho_{u}$ and $\rho_{d}$ are given in Table 1 for the number of quantization bits $b \leq 5$. For $b>5, \rho_{u}$ and $\rho_{d}$ can be approximated by $\rho_{u}\left(\rho_{d}\right)=\frac{\pi \sqrt{3}}{2} \cdot 2^{-2 b}[14,15] . \mathbf{q}_{U}$ and $\mathbf{q}_{D}$ denote the additive Gaussian quantization noise (AGQN) for uplink and downlink signals, respectively. For given channel matrices $\mathbf{G}_{a}(a \in\{D, U\}), \mathbf{G}_{L I}, \mathbf{G}_{I U}$, and precoding matrix $\mathbf{W}$, the covariance matrices of $\mathbf{q}_{U}$ and $\mathbf{q}_{D}$ are respectively given by [10]

$$
\begin{aligned}
\mathbf{R}_{\mathbf{q}_{U}=\mathbb{E}\left\{\mathbf{q}_{U} \mathbf{q}_{U}^{H}\right\}}=\alpha_{u}\left(1-\alpha_{u}\right) \operatorname{diag} \\
\left(P_{U}\left(\hat{\mathbf{G}}_{U}+\Delta \mathbf{G}_{U}\right)\left(\hat{\mathbf{G}}_{U}+\Delta \mathbf{G}_{U}\right)^{H}+\mathbf{T}\right),
\end{aligned}
$$

$$
\mathbf{R}_{\mathbf{q}_{D}}=\mathbb{E}\left\{\mathbf{q}_{D} \mathbf{q}_{D}^{H}\right\}=\alpha_{d}\left(1-\alpha_{d}\right) \operatorname{diag}\left(\mathbf{W} \mathbf{W}^{H}\right),
$$

where $\mathbf{T}=\eta P_{D} \mathbf{G}_{L I}\left(\alpha_{d}^{2} \mathbf{W} \mathbf{W}^{H}+\mathbf{R}_{\mathbf{q}_{D}}\right) \mathbf{G}_{L I}^{H}+\sigma_{U}^{2} \mathbf{I}_{M}$.

Table $1 \rho_{u}\left(\rho_{d}\right)$ for different ADC quantization bits $b(b \leq 5)$

\begin{tabular}{llllll}
\hline $\mathrm{b}$ & 1 & 2 & 3 & 4 & 5 \\
\hline$\rho_{u}\left(\rho_{d}\right)$ & 0.3634 & 0.1175 & 0.03454 & 0.009497 & 0.002499
\end{tabular}




\section{Analysis of uplink and downlink achievable rates}

The uplink and downlink asymptotic achievable rates for imperfect CSI are derived in this section. Then, we investigate that the quantization error, LI, and the IUI can be eliminated by applying power scaling law properly and increasing the number of antennas based on the derived expressions.

The maximal-ratio combining/maximum ratio transmission (MRC/MRT) are considered in the paper. The beamforming and precoding matrix of MRC/MRT are written as [16]

$$
\left\{\begin{array}{l}
\mathbf{F}=\hat{\mathbf{G}}_{U} \\
\mathbf{W}=\hat{\mathbf{G}}_{D}\left(\operatorname{tr}\left(\hat{\mathbf{G}}_{D}^{H} \hat{\mathbf{G}}_{D}\right)\right)^{-\frac{1}{2}},
\end{array}\right.
$$

where $\mathbf{F}$ and $\mathbf{W}$ denote the uplink decoding matrix at the receiver side and the downlink precoding matrix at the transmit side, respectively.

We first provide some results that will be used in the following derivations.

Lemma 1 The following results hold:

$$
\begin{aligned}
\mathbb{E}\left\{\left\|\hat{\mathbf{g}}_{a, n}\right\|^{2}\right\} & =\beta_{a, n} M \kappa_{a, n}, \\
\mathbb{E}\left\{\left|\hat{\mathbf{g}}_{a, n}^{H} \hat{\mathbf{g}}_{a, i}\right|^{2}\right\} & = \begin{cases}\beta_{a, n}^{2}\left(M+M^{2}\right) \kappa_{a, n}^{2}, & n=i \\
\beta_{a, n} \beta_{a, i} M \kappa_{a, n} \kappa_{a, i}, & n \neq i\end{cases}
\end{aligned}
$$

where $\kappa_{a, n}=\frac{P_{p} \beta_{a, n}}{1+P_{p} \beta_{a, n}}(a \in\{D, U\})$.

Proof The proof can be readily proved by using Lemma 3 in [17], when Rician $K$-factor is set as zero.

Lemma 2 The following results hold:

$$
\frac{1}{M} \hat{\mathbf{G}}_{a}^{H} \hat{\mathbf{G}}_{a} \rightarrow \hat{\mathbf{D}}_{a}
$$

where $\hat{\mathbf{D}}_{a}=\operatorname{diag}\left(\beta_{a, 1} \kappa_{a, 1}, \cdots, \beta_{a, n} \kappa_{a, n}, \cdots, \beta_{a, N} \kappa_{a, N}\right)$ is a diagonal matrix.

Proof The proof can be readily proved by using Lemma 5, (70) and (92) in [17], when Rician $K$-factor is set as zero.

\subsection{Analysis of uplink achievable rate}

With the help of (4) and (8), the quantized signal vector after the MRC receiver is processed as

$$
\mathbf{r}_{U}=\hat{\mathbf{G}}_{U}^{H} \tilde{\mathbf{y}}_{U}
$$

Then, the processed quantized signal vector $\mathbf{r}_{U}$ can be rewritten as

$$
\begin{aligned}
\mathbf{r}_{U}= & \alpha_{u} \sqrt{P_{U}} \hat{\mathbf{G}}_{U}^{H}\left(\hat{\mathbf{G}}_{U}+\Delta \mathbf{G}_{U}\right) \mathbf{x}_{U}+\alpha_{u} \hat{\mathbf{G}}_{U}^{H} \mathbf{n}_{U} \\
& +\hat{\mathbf{G}}_{U}^{H} \mathbf{q}_{U}+\alpha_{u} \sqrt{\eta} \sqrt{P_{D}} \hat{\mathbf{G}}_{U}^{H} \mathbf{G}_{L I}\left(\alpha_{d} \mathbf{W} \mathbf{x}_{D}+\mathbf{q}_{D}\right) .
\end{aligned}
$$

From (12), the received signal of $n$th user is given by

$$
\begin{aligned}
r_{U, n}= & \alpha_{u} \sqrt{P_{U}} \hat{\mathbf{g}}_{U, n}^{H} \hat{\mathbf{g}}_{U, n} x_{U, n} \\
& +\alpha_{u} \sqrt{P_{U}} \sum_{i=1, i \neq n}^{N} \hat{\mathbf{g}}_{U, n}^{H} \hat{\mathbf{g}}_{U, i} x_{U, i} \\
& +\alpha_{u} \sqrt{P_{U}} \sum_{i=1}^{N} \hat{\mathbf{g}}_{U, n}^{H} \Delta \mathbf{g}_{U, i} x_{U, i} \\
& +\alpha_{u} \alpha_{d} \sqrt{\eta} \sqrt{P_{D}} \sum_{i=1}^{N} \frac{\hat{\mathbf{g}}_{U, n}^{H} \mathbf{G}_{L I} \hat{\mathbf{g}}_{D, i}}{\sqrt{\operatorname{tr}\left(\mathbf{G}_{D}^{H} \mathbf{G}_{D}\right)}} x_{D, i} \\
& +\alpha_{u} \sqrt{\eta} \sqrt{P_{D}} \hat{\mathbf{g}}_{U, n}^{H} \mathbf{G}_{L I} \mathbf{q}_{D}+\alpha_{u} \hat{\mathbf{g}}_{U, n}^{H} \mathbf{n}_{U} \\
& +\hat{\mathbf{g}}_{U, n}^{H} \mathbf{q}_{U},
\end{aligned}
$$

where $\mathbf{g}_{U, n}$ is the $n$th column vector of $\mathbf{G}_{U}$.

With the help (13), the uplink achievable rate expression of the $n$th user with imperfect CSI is expressed as

$R_{U, n}=\frac{T-\tau}{T} \mathbb{E}\left\{\log _{2}\left(1+\frac{\left|A_{U, n}\right|^{2}}{\Psi_{U, n}+\alpha_{u}^{2}\left|\hat{\mathbf{g}}_{U, n}^{H}\right|^{2} \sigma_{U}^{2}}\right)\right\}$,

where

$$
A_{U, n}=\alpha_{u} \sqrt{P_{U}} \hat{\mathbf{g}}_{U, n}^{H} \hat{\mathbf{g}}_{U, n} x_{U, n},
$$

$\Psi_{U, n}=\left|B_{U, n}\right|^{2}+\left|C_{U, n}\right|^{2}+\left|D_{U, n}\right|^{2}+\left|E_{U, n}\right|^{2}+\left|F_{U, n}\right|^{2}$,

with

$$
\begin{aligned}
& B_{U, n}=\alpha_{u} \sqrt{P_{U}} \sum_{i=1, i \neq n}^{N} \hat{\mathbf{g}}_{U, n}^{H} \hat{\mathbf{g}}_{U, i} x_{U, i}, \\
& C_{U, n}=\alpha_{u} \sqrt{P_{U}} \sum_{i=1}^{N} \hat{\mathbf{g}}_{U, n}^{H} \Delta \mathbf{g}_{U, i} x_{U, i}, \\
& D_{U, n}=\alpha_{u} \alpha_{d} \sqrt{\eta} \sqrt{P_{D}} \sum_{i=1}^{N} \frac{\hat{\mathbf{g}}_{U, n}^{H} \mathbf{G}_{L I} \hat{\mathbf{g}}_{D, i}}{\sqrt{\operatorname{tr}\left(\hat{\mathbf{G}}_{D}^{H} \hat{\mathbf{G}}_{D}\right)}} x_{D, i}, \\
& E_{U, n}=\alpha_{u} \sqrt{\eta} \sqrt{P_{D}} \hat{\mathbf{g}}_{U, n}^{H} \mathbf{G}_{L I} \mathbf{q}_{D}, \\
& F_{U, n}=\hat{\mathbf{g}}_{U, n}^{H} \mathbf{q}_{U} .
\end{aligned}
$$

Theorem 1 For a massive MIMO system with a FD mode BS and multi-users, the uplink asymptotic achievable rate of the nth user based on MRC receivers for imperfect CSI can be derived as 
$R_{U, n} \approx \frac{T-\tau}{T} \log _{2}\left(1+\frac{\alpha_{u} P_{U} \beta_{U, n}(1+M) \kappa_{U, n}}{\Upsilon_{U, n}}\right)$

where $\Upsilon_{U, n}=P_{U} \sum_{i=1, i \neq n}^{N} \beta_{U, i} \kappa_{U, i}+\alpha_{d} \eta P_{D} \mu_{L I} M^{2}+$ $2\left(1-\alpha_{u}\right) P_{U} \beta_{U, n} \kappa_{U, n}+P_{U} \sum_{i=1}^{N} \Delta o_{U, i}^{2}+\sigma_{U}^{2}$.

Proof By using Lemma 1 in [17], we can approximate (14) as

$R_{U, n} \approx \frac{T-\tau}{T} \log _{2}\left(1+\frac{\mathbb{E}\left\{\left|A_{U, n}\right|^{2}\right\}}{\mathbb{E}\left\{\Psi_{U, n}\right\}+\alpha_{u}^{2} \mathbb{E}\left\{\left|\hat{\mathbf{g}}_{U, n}^{H}\right|^{2}\right\} \sigma_{U}^{2}}\right)$,

where

$$
\begin{gathered}
\mathbb{E}\left\{\left|A_{U, n}\right|^{2}\right\}=\alpha_{u}^{2} P_{U} \beta_{U, n}^{2}\left(M+M^{2}\right) \kappa_{U, n}^{2}, \\
\mathbb{E}\left\{\Psi_{U, n}\right\}=\mathbb{E}\left\{\left|B_{U, n}\right|^{2}\right\}+\mathbb{E}\left\{\left|C_{U, n}\right|^{2}\right\}+\mathbb{E}\left\{\left|D_{U, n}\right|^{2}\right\} \\
+\mathbb{E}\left\{\left|E_{U, n}\right|^{2}\right\}+\mathbb{E}\left\{\left|F_{U, n}\right|^{2}\right\},
\end{gathered}
$$

with

$$
\begin{aligned}
& \mathbb{E}\left\{\left|B_{U, n}\right|^{2}\right\}=\alpha_{u}^{2} P_{U} \sum_{i=1, i \neq n}^{N} \beta_{U, n} \beta_{U, i} M \kappa_{U, n} \kappa_{U, i}, \\
& \mathbb{E}\left\{\left|C_{U, n}\right|^{2}\right\}=\alpha_{U}^{2} P_{U} \sum_{i=1}^{N} \Delta o_{U, i}^{2} \beta_{U, n} M \kappa_{U, n}, \\
& \mathbb{E}\left\{\left|D_{U, n}\right|^{2}\right\}=\alpha_{u}^{2} \alpha_{d}^{2} \eta P_{D} \mu_{L I} \beta_{U, n} \kappa_{U, n} M^{2}, \\
& \mathbb{E}\left\{\left|E_{U, n}\right|^{2}\right\}=\alpha_{u}^{2} \alpha_{d}\left(1-\alpha_{d}\right) \eta P_{D} \mu_{L I} \beta_{U, n} M^{2} \kappa_{U, n}, \\
& \mathbb{E}\left\{\left|F_{U, n}\right|^{2}\right\} \\
& =2 \alpha_{u}\left(1-\alpha_{u}\right) P_{U} \beta_{U, n}^{2} M \kappa_{U, n}^{2} \\
& \quad+\alpha_{u}\left(1-\alpha_{u}\right) P_{U} \beta_{U, n} M \kappa_{U, n} \sum_{i=1, i \neq n}^{N} \beta_{U, i} \kappa_{U, i} \\
& \quad+\alpha_{u}\left(1-\alpha_{u}\right) P_{U} \beta_{U, n} M \kappa_{U, n} \sum_{i=1}^{N} \Delta o_{U, i}^{2} \\
& \quad+\alpha_{u}\left(1-\alpha_{u}\right)\left(\eta P_{D} \alpha_{d} \mu_{L I} \beta_{U, n} M^{2} \kappa_{U, n}+\beta_{U, n} M \kappa_{U, n} \sigma_{U}^{2}\right),
\end{aligned}
$$

$$
\begin{aligned}
\mathbb{E}\left\{\Psi_{U, n}\right\}= & \alpha_{u} P_{U} \sum_{i=1, i \neq n}^{N} \beta_{U, n} \beta_{U, i} M \kappa_{U, n} \kappa_{U, i} \\
& +\alpha_{u} \alpha_{d} \eta P_{D} \mu_{L I} \beta_{U, n} M^{2} \kappa_{U, n} \\
& +2 \alpha_{u}\left(1-\alpha_{u}\right) P_{U} \beta_{U, n}^{2} M \kappa_{U, n}^{2} \\
& +\alpha_{u} P_{U} \beta_{U, n} M \kappa_{U, n} \sum_{i=1}^{N} \Delta o_{U, i}^{2} \\
& +\alpha_{u}\left(1-\alpha_{u}\right) \beta_{U, n} M \kappa_{U, n} \sigma_{U}^{2} .
\end{aligned}
$$

See Appendix A for the detailed certification process.

Then, applying Lemma 1 and substituting (20), (27) into (19), (18) can be derived.

From (18), we analyze the effect of three factors on the rate performance, including the number of antennas $M$, the number of quantization bits, and the transmission power of BS and each user. In order to analyze Theorem 1 more intuitively, we analyze the following three approximate results, respectively.

Proposition 1 With fixed $P_{U}, P_{D}$, and $M$, when $b \rightarrow \infty$ $\left(\alpha_{u}=\alpha_{d}=1\right)$, then $R_{U, n}$ reduces to

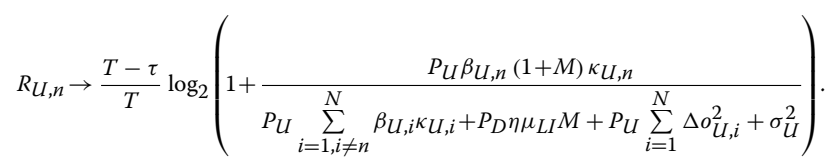

Equation (28) shows that the quantization error can be wiped out when the number of quantization bits $b$ tends to infinity. However, the effect of LI caused by FD mode BS cannot be eliminated. Given a fixed $M$, the uplink approximate achievable rate will converge to a constant. Therefore, the system performance that can be improved by increasing $A D C$ resolution is limited, implying that it is reasonable to adopt low-resolution ADCs/DACs in FD massive MIMO systems.

Proposition 2 With fixed $b$ and $M$, when $P_{U}=P_{D} \rightarrow$ $\infty, R_{U, n}$ converges to

$R_{U, n} \rightarrow \frac{T-\tau}{T} \log _{2}\left(1+\frac{\alpha_{u} \beta U, n(1+M) \kappa U, n}{\sum_{i=1, i \neq n}^{N} \beta_{U, i^{\kappa} U, i}+\alpha_{d} \eta \mu_{L I} M+2\left(1-\alpha_{U}\right) \beta_{U, n^{\kappa} U, n}+\sum_{i=1}^{N} \Delta o_{U, i}^{2}}\right)$,

which is consistent with Eq. (25) in [8] when the LI is eliminated completely and perfect CSI. Equation (29) shows that the rate depends not only on the number of antennas $M$ but also on the number of quantization bits $b$ when the transmission power of BS and users tend to infinity. Moreover, it is found that the quantization error and the effect 
of LI caused by FD mode BS neither can be ignored by increasing transmit power.

Proposition 3 Assuming that the transmission power of $B S$ and each user are scaled with $M$, i.e., $P_{U}=E_{U} / M$ and $P_{D}=E_{D} / M$, where $E_{U}$ and $E_{D}$ are fixed. When $M \rightarrow \infty$, $R_{U, n}$ tends to

$$
R_{U, n} \rightarrow \frac{T-\tau}{T} \log _{2}\left(1+\frac{\alpha_{u} E_{U} \beta_{U, n} \kappa_{U, n}}{\alpha_{d} \eta E_{D} \mu_{L I}+\sigma_{U}^{2}}\right)
$$

From (30), when the number of quantization bits $b \rightarrow$ $\infty, \sigma_{U}^{2}=1$, the LI is eliminated completely, and we can obtain $R_{U, n} \rightarrow \log _{2}\left(1+\alpha_{u} E_{U} \beta_{U, n}\right)$ under perfect CSI, which is consistent with Eq. (26) in [8]. The approximate uplink rate only relies on the number of quantization bits when $M$ tends to infinity.

\subsection{Analysis of downlink achievable rate}

With the help of (1), (3), (5), and (8), we can obtain the downlink received signal at the users

$$
\begin{aligned}
\mathbf{r}_{D}= & \sqrt{P_{D}}\left(\hat{\mathbf{G}}_{D}^{H}+\Delta \mathbf{G}_{D}^{H}\right) \tilde{\mathbf{y}}_{D}+\sqrt{P_{U}} \mathbf{G}_{I U, n}^{H} \mathbf{x}_{U}+\mathbf{n}_{D} \\
= & \sqrt{P_{D}} \hat{\mathbf{G}}_{D}^{H}\left(\alpha_{d} \mathbf{W} \mathbf{x}_{D}+\mathbf{q}_{D}\right)+\sqrt{P_{D}} \Delta \mathbf{G}_{D}^{H}\left(\alpha_{d} \mathbf{W} \mathbf{x}_{D}+\mathbf{q}_{D}\right) \\
& +\sqrt{P_{U}} \mathbf{G}_{I U, n}^{H} \mathbf{x}_{U}+\mathbf{n}_{D} .
\end{aligned}
$$

From (31), the received signal of $n$th user is given by

$$
\begin{aligned}
r_{D, n}= & \alpha_{d} \sqrt{P_{D}} \frac{\hat{\mathbf{g}}_{D, n}^{H} \hat{\mathbf{g}}_{D, n}}{\sqrt{\operatorname{tr}\left(\hat{\mathbf{G}}_{D}^{H} \hat{\mathbf{G}}_{D}\right)}} x_{D, n} \\
& +\alpha_{d} \sqrt{P_{D}} \sum_{i=1, i \neq n}^{N} \frac{\hat{\mathbf{g}}_{D, n}^{H} \hat{\mathbf{g}}_{D, i}}{\sqrt{\operatorname{tr}\left(\hat{\mathbf{G}}_{D}^{H} \hat{\mathbf{G}}_{D}\right)}} x_{D, i}+\sqrt{P_{D}} \hat{\mathbf{g}}_{D, n}^{H} \mathbf{q}_{D} \\
& +\alpha_{d} \sqrt{P_{D}} \sum_{i=1}^{N} \frac{\Delta \mathbf{g}_{D, n}^{H} \hat{\mathbf{g}}_{D, i}}{\sqrt{\operatorname{tr}\left(\hat{\mathbf{G}}_{D}^{H} \hat{\mathbf{G}}_{D}\right)}} x_{D, n}+\sqrt{P_{D}} \Delta \mathbf{g}_{D, n}^{H} \mathbf{q}_{D} \\
& +\sqrt{P_{U}} \mathbf{g}_{I U, n}^{H} \mathbf{x}_{U}+n_{D, n} .
\end{aligned}
$$

With the help of (32), the downlink achievable rate of the $n$th user is expressed as

$$
R_{D, n}=\frac{T-\tau}{T} \mathbb{E}\left\{\log _{2}\left(1+\frac{\left|A_{D, n}\right|^{2}}{\Psi_{D, n}+\sigma_{D, n}^{2}}\right)\right\},
$$

where

$$
\begin{gathered}
A_{D, n}=\alpha_{d} \sqrt{P_{D}} \frac{\hat{\mathbf{g}}_{D, n}^{H} \hat{\mathbf{g}}_{D, n}}{\sqrt{\operatorname{tr}\left(\hat{\mathbf{G}}_{D}^{H} \hat{\mathbf{G}}_{D}\right)}} x_{D, n}, \\
\Psi_{D, n}=\left|B_{D, n}\right|^{2}+\left|C_{D, n}\right|^{2}+\left|D_{D, n}\right|^{2}+\left|E_{D, n}\right|^{2}+\left|F_{D, n}\right|^{2},
\end{gathered}
$$

with

$$
\begin{aligned}
& B_{D, n}=\alpha_{d} \sqrt{P_{D}} \sum_{i=1, i \neq n}^{N} \frac{\hat{\mathbf{g}}_{D, n}^{H} \hat{\mathbf{g}}_{D, i}}{\sqrt{\operatorname{tr}\left(\hat{\mathbf{G}}_{D}^{H} \hat{\mathbf{G}}_{D}\right)}} x_{D, i}, \\
& C_{D, n}=\sqrt{P_{D}} \hat{\mathbf{g}}_{D, n}^{H} \mathbf{q}_{D}, \\
& D_{D, n}=\alpha_{d} \sqrt{P_{D}} \sum_{i=1}^{N} \frac{\Delta \mathbf{g}_{D, n}^{H} \hat{\mathbf{g}}_{D, i}}{\sqrt{\operatorname{tr}\left(\hat{\mathbf{G}}_{D}^{H} \hat{\mathbf{G}}_{D}\right)}} x_{D, n}, \\
& E_{D, n}=\sqrt{P_{D}} \Delta \mathbf{g}_{D, n}^{H} \mathbf{q}_{D}, \\
& F_{D, n}=\sqrt{P_{U}} \mathbf{g}_{I U, n}^{H} \mathbf{x}_{U} .
\end{aligned}
$$

Theorem 2 For a massive MIMO system with a FD mode BS and multi-users, the downlink asymptotic achievable rate of the nth user based on MRT processing for imperfect CSI can be derived as

$R_{D, n} \approx \frac{T-\tau}{T} \log _{2}\left(1+\frac{\alpha_{d}^{2} P_{D} \beta_{D, n}^{2}(M+1) \kappa_{D, n}^{2}}{\alpha_{d}^{2} P_{D} \mathrm{X}_{1}+P_{D} \mathrm{X}_{2}+\left(\alpha_{d} P_{D} \Delta o_{D, n}^{2}+\mathrm{X}_{3}\right) \operatorname{tr}\left(\hat{\mathbf{D}}_{D}\right)}\right)$,

where $\mathrm{X}_{1}=\beta_{D, n} \kappa_{D, n} \sum_{i=1, i \neq n}^{N} \beta_{D, i} \kappa_{D, i}, \mathrm{X}_{2}=\alpha_{d}\left(1-\alpha_{d}\right)$ $\beta_{D, n} \kappa_{D, n}\left(\beta_{D, n} \kappa_{D, n}+\sum_{i=1}^{N} \beta_{D, i} \kappa_{D, i}\right), \mathrm{X}_{3}=P_{U} \sum_{i=1}^{N} \sigma_{i n}+\sigma_{D, n}^{2}$.

Proof By using Lemma 1 in [17], we can approximate (33) as

$$
R_{U, n} \approx \frac{T-\tau}{T} \log _{2}\left(1+\frac{\mathbb{E}\left\{\left|A_{D, n}\right|^{2}\right\}}{\mathbb{E}\left\{\Psi_{D, n}\right\}+\sigma_{D, n}^{2}}\right),
$$

where

$$
\begin{aligned}
\mathbb{E}\left\{\left|A_{D, n}\right|^{2}\right\} & =\alpha_{d}^{2} P_{D} \beta_{D, n}^{2}(M+1) \kappa_{D, n}^{2}\left(\operatorname{tr}\left(\hat{\mathbf{D}}_{D}\right)\right)^{-1}, \\
\mathbb{E}\left\{\Psi_{D, n}\right\}= & \mathbb{E}\left\{\left|B_{D, n}\right|^{2}\right\}+\mathbb{E}\left\{\left|C_{D, n}\right|^{2}\right\}+\mathbb{E}\left\{\left|D_{D, n}\right|^{2}\right\} \\
& +\mathbb{E}\left\{\left|E_{D, n}\right|^{2}\right\}+\mathbb{E}\left\{\left|F_{D, n}\right|^{2}\right\},
\end{aligned}
$$

with

$\mathbb{E}\left\{\left|B_{D, n}\right|^{2}\right\}=\alpha_{d}^{2} P_{D} \beta_{D, n} \beta_{D, i} M \kappa_{D, n} \kappa_{D, i}\left(\operatorname{tr}\left(\hat{\mathbf{D}}_{D}\right)\right)^{-1}$,

$$
\begin{aligned}
\mathbb{E}\left\{\left|C_{D, n}\right|^{2}\right\} \\
=2 P_{D} \alpha_{d}\left(1-\alpha_{d}\right) \beta_{D, n}^{2} \kappa_{D, n}^{2}\left(\operatorname{tr}\left(\hat{\mathbf{D}}_{D}\right)\right)^{-1} \\
\quad+P_{D} \alpha_{d}\left(1-\alpha_{d}\right) \beta_{D, n} \kappa_{D, n} \sum_{i=1, i \neq n}^{N} \beta_{D, i} \kappa_{D, i}\left(\operatorname{tr}\left(\hat{\mathbf{D}}_{D}\right)\right)^{-1}
\end{aligned}
$$




$$
\begin{gathered}
\mathbb{E}\left\{\left|D_{U, n}\right|^{2}\right\}=\alpha_{d}^{2} P_{D} \Delta o_{D, n}^{2}, \\
\mathbb{E}\left\{\left|E_{U, n}\right|^{2}\right\}=\alpha_{d}\left(1-\alpha_{d}\right) P_{D} \Delta o_{D, n}^{2}, \\
\mathbb{E}\left\{\left|F_{U, n}\right|^{2}\right\}=P_{U} \sum_{i=1}^{N} \sigma_{i n} .
\end{gathered}
$$

See Appendix B for the detailed certification process. Substituting (39), (40) into (38), (37) can be derived.

Similarly, we analyze the effect of three factors on the downlink rate performance, including the number of antennas $M$, the number of quantization bits $b$ and the transmission power of BS and each user.

Proposition 4 With fixed $P_{U}, P_{D}$, and $M$, when $b \rightarrow \infty$ $\left(\alpha_{d}=1\right), R_{D, n}$ reduces to

$$
R_{D, n} \rightarrow \frac{T-\tau}{T} \log _{2}\left(1+\frac{P_{D} \beta_{D, n}^{2}(1+M) \kappa_{D, n}^{2}}{P_{D} \mathrm{X}_{1}+\left(P_{D} \Delta o_{D, n}^{2}+\mathrm{X}_{3}\right) \operatorname{tr}\left(\hat{\mathbf{D}}_{D}\right)}\right)
$$

Equation(46) shows that the quantization error can be wiped out when the number of quantization bits $b$ tends to infinity. Given a fixed $M$, the downlink approximate achievable rate will converge to a constant. Therefore, the system performance that can be improved by increasing $D A C$ resolution is limited.

Proposition 5 With fixed $b$ and $M$, when $P_{U}=P_{D} \rightarrow \infty$, $R_{D, n}$ converges to

$$
R_{D, n} \rightarrow \frac{T-\tau}{T} \log _{2}\left(1+\frac{\alpha_{d}^{2} \beta_{D, n}^{2}(1+M) \kappa_{D, n}^{2}}{\alpha_{d}^{2} \mathrm{X}_{1}+\mathrm{X}_{2}+\left(\alpha_{d} P_{D} \Delta o_{D, n}^{2}+\sum_{i=1}^{N} \sigma_{i n}\right) \operatorname{tr}\left(\hat{\mathbf{D}}_{D}\right)}\right) .
$$

From (47), we can see that the rate depends not only on the number of antennas $M$ but also on the number of quantization bits $b$ when the transmission power of BS and users tend to infinity. Moreover, it is found that the quantization error and the effect of IUI caused by FD mode users neither can be ignored by increasing transmit power.

Proposition 6 Assuming that the transmission power of $B S$ and each user are scaled with $M$, i.e., $P_{U}=E_{U} / M$ and $P_{D}=E_{D} / M$, where $E_{U}$ and $E_{D}$ are fixed. When $M \rightarrow \infty$, $R_{U, n}$ tends to

$$
R_{D, n} \rightarrow \frac{T-\tau}{T} \log _{2}\left(1+\frac{\alpha_{d}^{2} E_{D} \beta_{D, n}^{2} \kappa_{D, n}^{2}}{\sigma_{D, n}^{2} \operatorname{tr}\left(\hat{\mathbf{D}}_{D}\right)}\right)
$$

It is found that the IUI caused by FD can be eliminated by applying power scaling law properly and increasing the number of antennas $M$. The approximate downlink rate only relies on the number of quantization bits when $M$ tends to infinity.

\section{Simulation results}

In this section, we analyze the simulation results of uplink and downlink sum achievable rates of the system model proposed in this paper by building a simulation environment where users uniformly distributed in a cell with a radius of $1000 \mathrm{~m}$. Moreover, the sum rate of this system are defined as $C_{\text {sum }}=C_{U}+C_{D}$ [18], where $C_{U}$ and $C_{D}$ are the uplink and downlink sum achievable rates of the system, respectively. Assuming the minimum distance from the user to the BS is $r_{\min }=100 \mathrm{~m}$, and $r_{n}$ is the distance from the $n$th user to the BS. $a_{n}$ is denoted as a log-normal random variable with standard deviation $\sigma=8 \mathrm{~dB} . v=$ 3.8 is the path loss exponent. The large-scale channel fading can be modeled as $\beta_{n}=a_{n}\left(r_{n} / r_{\text {min }}\right)^{-v}$, [19-21]. In this simulation, we assume that the number of users is $N=10$, the variances of the noise are $\sigma_{D}=\sigma_{U}=1$, and the residual LI power is $\rho=\beta_{L I}=0 \mathrm{~dB}, P_{U}=10 \mathrm{~dB}$ and $P_{D}=N P_{U}$. The length of channel coherence time is set to $T=300$ (symbols), and the length of pilot sequence is set to $\tau=2 N$.

The FD sum achievable rate versus the different numbers of antennas at the BS is given in Fig. 2. Results are simulated for the three different numbers of quantization bits $b$ with 1,2 , and $\infty$. The gap between these curves decreases as $b$ increases which means the performance that is improved by increasing the number of quantization bits $b$ is quite finite. This proves that it is reasonable to adopt low-resolution ADCs in FD massive MIMO systems.

Figure 3 presents the uplink sum rate of the proposed MIMO system with or without power scaling versus different residual LI power $\eta \mu_{L I}$ when $M=200$. As shown in Fig. 3, the uplink sum rate is constant when the residual LI power $\eta \mu_{L I}$ is small. As the residual LI power gradually increases, the uplink sum rate decreases and the performance of the proposed system deteriorates.

Figures 4 and 5 depict the uplink and downlink sum achievable rates versus different the number of quantization bits $b$, respectively. In this simulation, we choose the number of antennas $M=100$. As shown in Figs. 4 and 5, the uplink and downlink rates increase with the number of quantization bits and converge to a constant which can be obtained by (28) and (46) when the number of quantization bits $b>5$. The figures demonstrate the rationality 


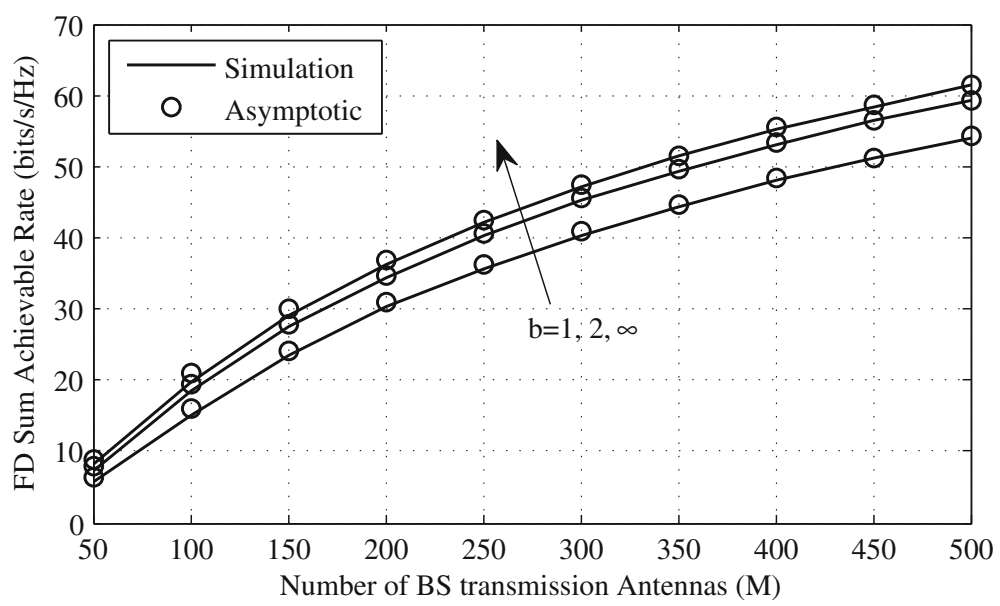

Fig. 2 FD sum rate for different $b$. Comparison of FD sum rate for the different numbers of quantization bits $b$ versus the number of antennas at the BS

of adopting low-resolution ADCs/DACs in FD massive MIMO systems.

\section{Conclusions}

A FD massive MIMO system where low-resolution ADCs/DACs are equipped in the receive antennas and transmit antennas at the FD mode $\mathrm{BS}$ is investigated in this paper. With the help of MRC/MRT processing and MMSE, the approximate uplink and downlink rates for imperfect CSI have been derived in this paper. The theoretical results reveal that the loss of system performance due to the quantization error of the low-resolution ADCs and LI can be compensated by applying power scaling law properly and increasing the number of antennas. This point turns out to be reasonable to adopt low-resolution $\mathrm{ADCs} / \mathrm{DACs}$ in FD massive MIMO systems.

\section{Appendix A Proof of Theorem 1}

For calculating (19), where $A_{U, n}$ is the desired signal, $B_{U, n}$ is the multi-user interference, $C_{U, n}$ is the LI, $D_{U, n}$ is the AGQN at the transmit antennas of the BS, $E_{U, n}$ is the AWGN, and $F_{U, n}$ is the AGQN at the receive antennas of the BS, we compute them one by one as follows.

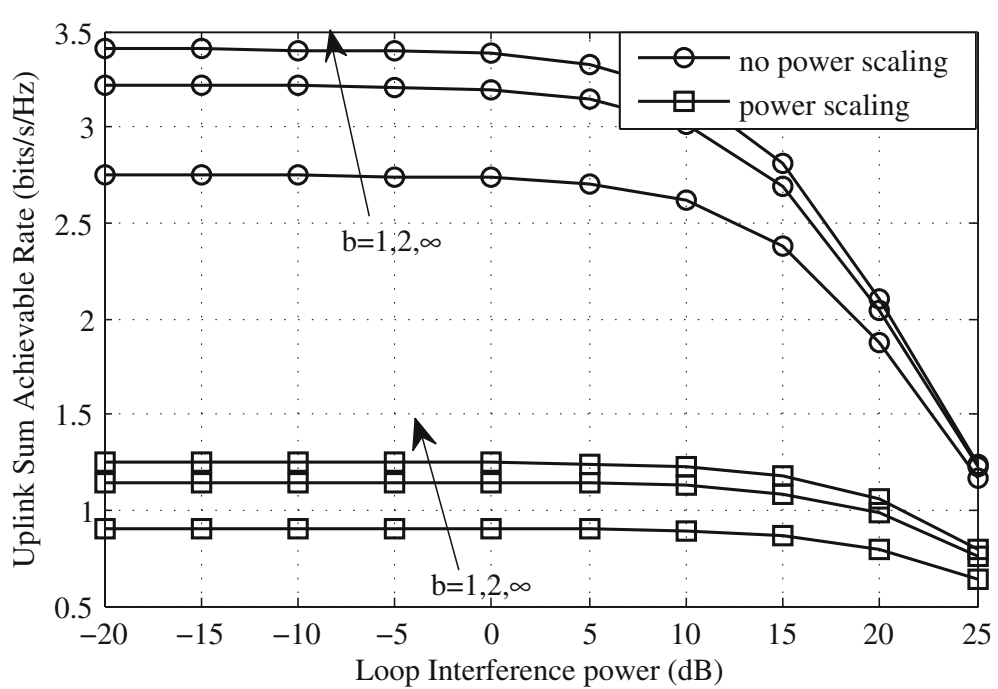

Fig. 3 Uplink sum achievable rate for power scaling and no power scaling. Comparison of uplink sum rate for the different numbers of quantization bits $b$ versus the residual LI power $\eta \mu_{L I}$ 


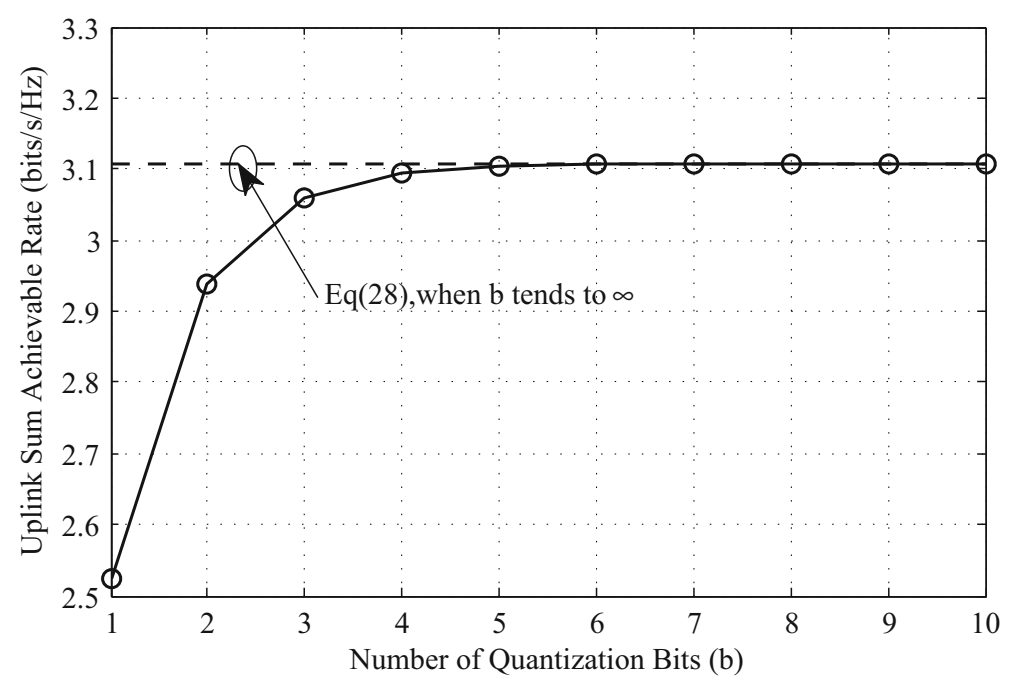

Fig. 4 Uplink sum achievable rate when $b$ tends to be $\infty$. Description of uplink sum rate for the different numbers of quantization bits $b$ and $b$ tends to be $\infty$ which can be obtained by (28)

1. Compute $\mathbb{E}\left\{\left|A_{U, n}\right|^{2}\right\}$ :

$$
\begin{aligned}
\mathbb{E}\left\{\left|A_{U, n}\right|^{2}\right\} & =\alpha_{u}^{2} P_{U} \mathbb{E}\left\{\left|\hat{\mathbf{g}}_{U, n}^{H} \hat{\mathbf{g}}_{U, n}\right|^{2}\right\} \\
& =\alpha_{u}^{2} P_{U} \beta_{U, n}^{2}\left(M^{2}+M\right) \kappa_{U, n}^{2}
\end{aligned}
$$

2. Compute $\mathbb{E}\left\{\left|B_{U, n}\right|^{2}\right\}$ :

$$
\begin{aligned}
\mathbb{E}\left\{\left|B_{U, n}\right|^{2}\right\} & =\alpha_{u}^{2} P_{U} \sum_{i=1, i \neq n}^{N} \mathbb{E}\left\{\left|\hat{\mathbf{g}}_{U, n}^{H} \hat{\mathbf{g}}_{U, i}\right|^{2}\right\} \\
& =\alpha_{u}^{2} P_{U} \sum_{i=1, i \neq n}^{N} \beta_{U, n} \beta_{U, i} M \kappa_{U, n} \kappa_{U, i}
\end{aligned}
$$

(50)
3. Compute $\mathbb{E}\left\{\left|C_{U, n}\right|^{2}\right\}$ :

$$
\begin{aligned}
\mathbb{E}\left\{\left|C_{U, n}\right|^{2}\right\} & =\alpha_{u}^{2} P_{U} \sum_{i=1}^{N} \mathbb{E}\left\{\left|\hat{\mathbf{g}}_{U, n}^{H} \Delta \mathbf{g}_{U, i}\right|^{2}\right\} \\
& =\alpha_{u}^{2} P_{U} \sum_{i=1}^{N} \mathbb{E}\left\{\hat{\mathbf{g}}_{U, n}^{H} \Delta \mathbf{g}_{U, i} \Delta \mathbf{g}_{U, i}^{H} \hat{\mathbf{g}}_{U, n}\right\} \\
& =\alpha_{u}^{2} P_{U} \sum_{i=1}^{N} \mathbb{E}\left\{\hat{\mathbf{g}}_{U, n}^{H} o_{U, i}^{2} \hat{\mathbf{g}}_{U, n}\right\} \\
& =\alpha_{u}^{2} P_{U} \beta_{U, n} M \kappa_{U, n} \sum_{i=1}^{N} o_{U, i}^{2}
\end{aligned}
$$

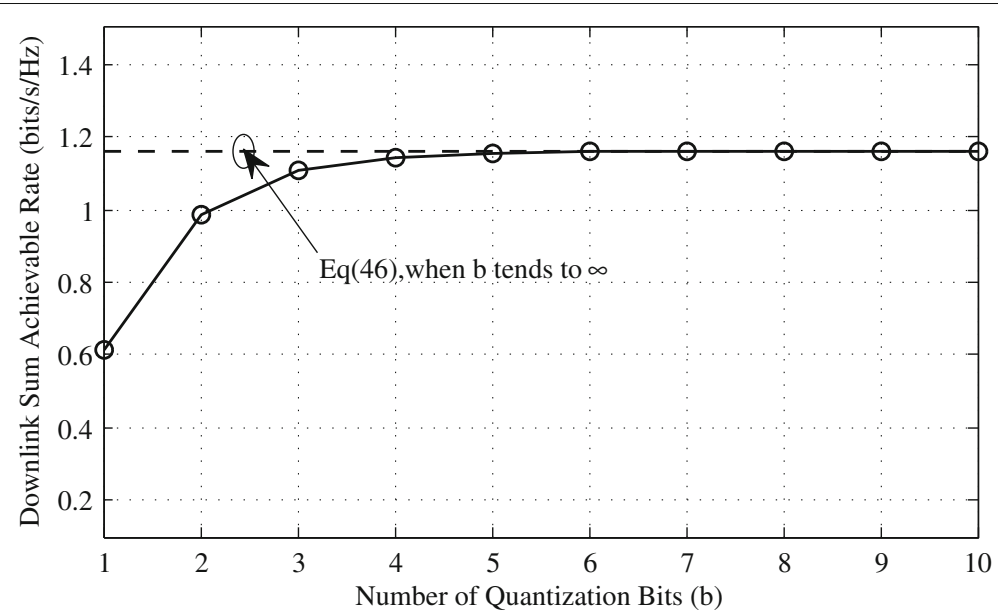

Fig. 5 Downlink sum achievable rate when $b$ tends to be $\infty$. Description of downlink sum rate for the different numbers of quantization bits $b$ and $b$ tends to be $\infty$ which can be obtained by (46) 
4. Compute $\mathbb{E}\left\{\left|D_{U, n}\right|^{2}\right\}$ :

$$
\begin{aligned}
\mathbb{E}\left\{\left|D_{U, n}\right|^{2}\right\} & =\alpha_{u}^{2} \alpha_{d}^{2} \eta P_{D} \sum_{i=1}^{N} \frac{\mathbb{E}\left\{\left|\hat{\mathbf{g}}_{U, n}^{H} \mathbf{G}_{L I} \hat{\mathbf{g}}_{D, i}\right|^{2}\right\}}{\operatorname{tr}\left(\hat{\mathbf{G}}_{D}^{H} \hat{\mathbf{G}}_{D}\right)} \\
& =\alpha_{u}^{2} \alpha_{d}^{2} \eta P_{D} \frac{1}{M} \sum_{i=1}^{N} \mathbb{E}\left\{\hat{\mathbf{g}}_{U, n}^{H} \mathbf{G}_{L I} \hat{\mathbf{g}}_{D, i} \hat{\mathbf{g}}_{D, i}^{H} \mathbf{G}_{L I}^{H} \hat{\mathbf{g}}_{U, n}\right\}\left(\operatorname{tr}\left(\frac{\hat{\mathbf{G}}_{D}^{H} \hat{\mathbf{G}}_{D}}{M}\right)\right)^{-1} \\
& =\alpha_{u}^{2} \alpha_{d}^{2} \eta P_{D} \frac{1}{M} \sum_{i=1}^{N} \mathbb{E}\left\{\hat{\mathbf{g}}_{U, n}^{H} \mathbf{G}_{L I} \beta_{D, i} M \kappa_{D, i} \mathbf{G}_{L I}^{H} \hat{\mathbf{g}}_{U, n}\right\}\left(\operatorname{tr}\left(\hat{\mathbf{D}}_{D}\right)\right)^{-1} \\
& =\alpha_{u}^{2} \alpha_{d}^{2} \eta P_{D} \mu_{L I} M \sum_{i=1}^{N} \beta_{D, i} \kappa_{D, i} \mathbb{E}\left\{\hat{\mathbf{s}}_{U, n}^{H} \hat{\mathbf{g}}_{U, n}\right\}\left(\operatorname{tr}\left(\hat{\mathbf{D}}_{D}\right)\right)^{-1} \\
& =\alpha_{u}^{2} \alpha_{d}^{2} \eta P_{D} \mu_{L I} \beta_{U, n} M^{2} \kappa_{U, n}
\end{aligned}
$$

5. Compute $\mathbb{E}\left\{\left|E_{U, n}\right|^{2}\right\}$ :

$$
\mathbb{E}\left\{\mathbf{q}_{D} \mathbf{q}_{D}^{H}\right\}=\alpha_{d}\left(1-\alpha_{d}\right) \operatorname{diag}\left(\mathbf{G}_{D} \mathbf{G}_{D}^{H}\right)\left(\operatorname{tr}\left(\mathbf{G}_{D} \mathbf{G}_{D}^{H}\right)\right)^{-1}
$$

$$
\begin{aligned}
\mathbb{E}\left\{\left|E_{U, n}\right|^{2}\right\} & =\alpha_{u}^{2} \eta P_{D} \mathbb{E}\left\{\left|\mathbf{g}_{U, n}^{H} \mathbf{G}_{L I} \mathbf{q}_{D}\right|^{2}\right\} \\
& =\alpha_{u}^{2} \eta P_{D} \mathbb{E}\left\{\mathbf{g}_{U, n}^{H} \mathbf{G}_{L I} \mathbf{q}_{D} \mathbf{q}_{D}^{H} \mathbf{G}_{L I}^{H} \mathbf{g}_{U, n}\right\} \\
& =\alpha_{u}^{2} \alpha_{d}\left(1-\alpha_{d}\right) \eta P_{D} \mathbb{E}\left\{\mathbf{g}_{U, n}^{H} \mathbf{G}_{L I} \operatorname{diag}\left(\hat{\mathbf{G}}_{D} \hat{\mathbf{G}}_{D}^{H}\right) \mathbf{G}_{L I}^{H} \mathbf{g}_{U, n}\right\}\left(\operatorname{tr}\left(\hat{\mathbf{G}}_{D}^{H} \hat{\mathbf{G}}_{D}\right)\right)^{-1} \\
& =\alpha_{u}^{2} \alpha_{d}\left(1-\alpha_{d}\right) \eta P_{D} \mathbb{E}\left\{\mathbf{g}_{U, n}^{H} \mathbf{G}_{L I} \operatorname{diag}\left(\frac{\hat{\mathbf{G}}_{D} \hat{\mathbf{G}}_{D}^{H}}{M}\right) \mathbf{G}_{L I}^{H} \mathbf{g}_{U, n}\right\}\left(\operatorname{tr}\left(\frac{\hat{\mathbf{G}}_{D}^{H} \hat{\mathbf{G}}_{D}}{M}\right)\right)^{-1} \\
& =\alpha_{u}^{2} \alpha_{d}\left(1-\alpha_{d}\right) \eta P_{D} \mathbb{E}\left\{\mathbf{g}_{U, n}^{H} \mathbf{G}_{L I} \hat{\mathbf{D}}_{D} \mathbf{G}_{L I}^{H} \mathbf{g}_{U, n}\right\}\left(\operatorname{tr}\left(\hat{\mathbf{D}}_{D}\right)\right)^{-1} \\
& =\alpha_{u}^{2} \alpha_{d}\left(1-\alpha_{d}\right) \eta P_{D} \mu_{L I} \beta_{U, n} M^{2} \kappa U, n
\end{aligned}
$$

6. Compute $\mathbb{E}\left\{\left|F_{U, n}\right|^{2}\right\}$ :

$$
\begin{aligned}
\mathbb{E}\left\{\left|F_{U, n}\right|^{2}\right\}= & \mathbb{E}\left\{\left|\hat{\mathbf{g}}_{U, n}^{H} \mathbf{q}_{U}\right|^{2}\right\} \\
= & \alpha_{U}\left(1-\alpha_{U}\right) \mathbb{E}\left\{\hat{\mathbf{g}}_{U, n}^{H} \operatorname{diag}\left(P_{U}\left(\hat{\mathbf{G}}_{U}+\Delta \mathbf{G}_{U}\right)\left(\hat{\mathbf{G}}_{U}+\Delta \mathbf{G}_{U}\right)^{H}+\mathbf{T}\right) \hat{\mathbf{g}}_{U, n}\right\} \\
= & \alpha_{U}\left(1-\alpha_{U}\right) \mathbb{E}\left\{\hat{\mathbf{g}}_{U, n}^{H} \operatorname{diag}\left(P_{U} \hat{\mathbf{G}}_{U} \hat{\mathbf{G}}_{U}^{H}+\Delta \mathbf{G}_{U} \Delta \mathbf{G}_{U}^{H}+\mathbf{T}\right) \hat{\mathbf{g}}_{U, n}\right\} \\
= & \alpha_{U}\left(1-\alpha_{U}\right) \mathbb{E}\left\{\hat{\mathbf{g}}_{U, n}^{H} \operatorname{diag}\left(P_{U} \mathbf{G}_{U} \mathbf{G}_{U}^{H}\right) \hat{\mathbf{g}}_{U, n}\right\} \\
& +\alpha_{U}\left(1-\alpha_{U}\right) \mathbb{E}\left\{\hat{\mathbf{g}}_{U, n}^{H} \operatorname{diag}\left(\Delta \mathbf{G}_{U} \Delta \mathbf{G}_{U}^{H}\right) \hat{\mathbf{g}}_{U, n}\right\} \\
& +\alpha_{U}\left(1-\alpha_{U}\right)\left\{\hat{\mathbf{g}}_{U, n}^{H} \operatorname{diag}(\mathbf{T}) \hat{\mathbf{g}}_{U, n}\right\}
\end{aligned}
$$

We can derive the first term in (55) as:

$$
\begin{array}{r}
\alpha_{u}\left(1-\alpha_{u}\right) \mathbb{E}\left\{\hat{\mathbf{g}}_{U, n}^{H} \operatorname{diag}\left(P_{U} \hat{\mathbf{G}}_{U} \hat{\mathbf{G}}_{U}^{H}\right) \hat{\mathbf{g}}_{U, n}\right\}=\alpha_{u}\left(1-\alpha_{u}\right) \\
P_{U}\left(2 M \beta_{U, n}^{2} \kappa_{U, n}^{2}+M \beta_{U, n} \kappa_{U, n} \sum_{i=1, i \neq n}^{N} \beta_{U, i} \kappa_{U, i}\right)
\end{array}
$$

The second term in (55) can be derived as

$$
\begin{array}{r}
\alpha_{u}\left(1-\alpha_{u}\right) \mathbb{E}\left\{\mathbf{g}_{U, n}^{H} \operatorname{diag}\left(\Delta \mathbf{G}_{U} \Delta \mathbf{G}_{U}^{H}\right) \mathbf{g}_{U, n}\right\} \\
=\alpha_{u}\left(1-\alpha_{u}\right) \beta_{U, n} M \kappa_{U, n} \sum_{i=1}^{N} \Delta o_{U, i}^{2}
\end{array}
$$

The third term in (55) can be derived as

$$
\begin{aligned}
\alpha_{u}\left(1-\alpha_{u}\right) \mathbb{E}\{ & \left.\mathbf{g}_{U, n}^{H} \operatorname{diag}(\mathbf{T}) \mathbf{g}_{U, n}\right\} \\
= & \alpha_{u}\left(1-\alpha_{u}\right) \mathbb{E}\left\{\mathbf{g}_{U, n}^{H} \operatorname{diag}\left(\eta P_{D} \alpha_{d}^{2} \mathbf{G}_{L I} \mathbf{W} \mathbf{W}^{H} \mathbf{G}_{L I}^{H}\right) \mathbf{g}_{U, n}\right\} \\
& +\alpha_{u}\left(1-\alpha_{u}\right) \mathbb{E}\left\{\mathbf{g}_{U, n}^{H} \operatorname{diag}\left(\eta P_{D} \mathbf{G}_{L I} \mathbf{q}_{D} \mathbf{q}_{D}^{H} \mathbf{G}_{L I}^{H}\right) \mathbf{g}_{U, n}\right\} \\
& +\alpha_{u}\left(1-\alpha_{u}\right) \mathbb{E}\left\{\mathbf{g}_{U, n}^{H} \sigma_{U}^{2} \mathbf{I}_{M} \mathbf{g}_{U, n}\right\} \\
= & \alpha_{u}\left(1-\alpha_{u}\right) \eta P_{D} \alpha_{d}^{2} \mu_{L I} \beta_{U, n} M^{2} \kappa_{U, n} \\
& +\alpha_{u}\left(1-\alpha_{u}\right) \eta P_{D} \alpha_{d}\left(1-\alpha_{d}\right) \mu_{L I} \beta_{U, n} M^{2} \kappa_{U, n} \\
& +\alpha_{u}\left(1-\alpha_{u}\right) \sigma_{U}^{2} \beta_{U, n} M \kappa_{U, n} \\
= & \alpha_{u}\left(1-\alpha_{u}\right) \alpha_{d} \eta P_{D} \mu_{L I} \beta_{U, n} M^{2} \kappa_{U, n} \\
& +\alpha_{u}\left(1-\alpha_{u}\right) \sigma_{U}^{2} \beta_{U, n} M \kappa_{U, n}
\end{aligned}
$$

Combine (56), (57), and (58), we have

$$
\begin{aligned}
\mathbb{E}\left\{\left|F_{U, n}\right|^{2}\right\}= & \alpha_{u}\left(1-\alpha_{U}\right) P_{U}\left(2 M \beta_{U, n}^{2} \kappa_{U, n}^{2}+M \beta_{U, n} \kappa U, n \sum_{i=1, i \neq n}^{N} \beta_{U, i} \kappa_{U, i}\right) \\
& +\alpha_{u}\left(1-\alpha_{u}\right) \beta_{U, n} M \kappa_{U, n} \sum_{i=1}^{N} \Delta o_{U, i}^{2} \\
& +\alpha_{u}\left(1-\alpha_{u}\right) \alpha_{d} \eta P_{D} \mu_{L I} \beta_{U, n} M^{2} \kappa_{U, n} \\
& +\alpha_{u}\left(1-\alpha_{u}\right) \sigma_{U}^{2} \beta_{U, n} M \kappa_{U, n}
\end{aligned}
$$

\section{Appendix B Proof of Theorem 2}

For calculating (38), where $A_{D, n}$ is the desired signal, $B_{D, n}$ is the multi-user interference, $C_{D, n}$ is the $\mathrm{LI}, D_{D, n}$ is the AGQN at the transmit antennas of the BS, $E_{D, n}$ is the $\mathrm{AWGN}$, and $F_{D, n}$ is the AGQN at the receive antennas of the BS, we compute them one by one as follows.

7. Compute $\mathbb{E}\left\{\left|A_{D, n}\right|^{2}\right\}$ :

$$
\begin{aligned}
\mathbb{E}\left\{\left|A_{D, n}\right|^{2}\right\} & =\alpha_{d}^{2} P_{D} \mathbb{E}\left\{\left|\hat{\mathbf{g}}_{D, n}^{H} \hat{\mathbf{g}}_{D, n}\right|^{2}\right\}\left(\operatorname{tr}\left(\hat{\mathbf{G}}_{D}^{H} \hat{\mathbf{G}}_{D}\right)\right)^{-1} \\
& =\alpha_{d}^{2} P_{D} \beta_{D, n}^{2}\left(M^{2}+M\right) \kappa_{D, n}^{2} \frac{1}{M}\left(\operatorname{tr}\left(\frac{\hat{\mathbf{G}}_{D}^{H} \hat{\mathbf{G}}_{D}}{M}\right)\right)^{-1} \\
& =\alpha_{d}^{2} P_{D} \beta_{D, n}^{2}(M+1) \kappa_{D, n}^{2}\left(\operatorname{tr}\left(\hat{\mathbf{D}}_{D}\right)\right)^{-1}
\end{aligned}
$$


8. Compute $\mathbb{E}\left\{\left|B_{D, n}\right|^{2}\right\}$ :

$$
\begin{aligned}
\mathbb{E}\left\{\left|B_{D, n}\right|^{2}\right\} & =\alpha_{d}^{2} P_{D} \sum_{i=1, i \neq n}^{N} \mathbb{E}\left\{\left|\hat{\mathbf{g}}_{D, n}^{H} \hat{\mathbf{g}}_{D, i}\right|^{2}\right\}\left(\operatorname{tr}\left(\hat{\mathbf{G}}_{D}^{H} \hat{\mathbf{G}}_{D}\right)\right)^{-1} \\
& =\alpha_{d}^{2} P_{D} \beta_{U, n} \kappa_{U, n} \sum_{i=1, i \neq n}^{N} \beta_{U, i} \kappa_{U, i}\left(\operatorname{tr}\left(\hat{\mathbf{D}}_{D}\right)\right)^{-1}
\end{aligned}
$$

9. Compute $\mathbb{E}\left\{\left|C_{D, n}\right|^{2}\right\}$ :

$$
\begin{aligned}
\mathbb{E}\left\{\left|C_{D, n}\right|^{2}\right\} & =P_{D} \mathbb{E}\left\{\left|\hat{\mathbf{g}}_{D, n}^{H} \mathbf{q}_{D}\right|^{2}\right\} \\
& =P_{D} \alpha_{d}\left(1-\alpha_{d}\right) \mathbb{E}\left\{\hat{\mathbf{g}}_{D, n}^{H} \operatorname{diag}\left(\hat{\mathbf{G}}_{D} \hat{\mathbf{G}}_{D}^{H}\right) \hat{\mathbf{g}}_{D, n}\right\}\left(\operatorname{tr}\left(\hat{\mathbf{G}}_{D} \hat{\mathbf{G}}_{D}^{H}\right)\right)^{-1} \\
& =P_{D} \alpha_{d}\left(1-\alpha_{d}\right)\left(2 M \beta_{D, n}^{2} \kappa_{D, n}^{2}+M \beta_{D, n} \kappa_{D, n} \sum_{i=1, i \neq n}^{N} \beta_{D, i} \kappa_{D, i}\right)\left(\operatorname{tr}\left(\frac{\hat{\mathbf{b}}_{D}}{M}\right)\right)^{-1} \\
& =P_{D} \alpha_{d}\left(1-\alpha_{d}\right)\left(2 \beta_{D, n}^{2} \kappa_{D, n}^{2}+\beta_{D, n} \kappa_{D, n} \sum_{i=1, i \neq n}^{N} \beta_{D, i \kappa}\right)\left(\operatorname{tr}\left(\hat{\mathbf{D}}_{D}\right)\right)^{-1}
\end{aligned}
$$

10. Compute $\mathbb{E}\left\{\left|D_{D, n}\right|^{2}\right\}$ :

$$
\begin{aligned}
\mathbb{E}\left\{\left|D_{D, n}\right|^{2}\right\} & =\alpha_{d}^{2} P_{D} \sum_{i=1}^{N} \mathbb{E}\left\{\left|\Delta \mathbf{g}_{D, n}^{H} \hat{\mathbf{g}}_{D, i}\right|^{2}\right\}\left(\operatorname{tr}\left(\hat{\mathbf{G}}_{D}^{H} \hat{\mathbf{G}}_{D}\right)\right)^{-1} \\
& =\alpha_{d}^{2} P_{D} \sum_{i=1}^{N} \beta_{D, i} \kappa_{D, i} M \Delta o_{D, n}^{2}\left(\operatorname{tr}\left(\frac{\hat{\mathbf{D}}_{D}}{M}\right)\right)^{-1} \\
& =\alpha_{d}^{2} P_{D} \Delta o_{D, n}^{2} \sum_{i=1}^{N} \beta_{D, i} \kappa_{D, i}\left(\operatorname{tr}\left(\hat{\mathbf{D}}_{D}\right)\right)^{-1} \\
& =\alpha_{d}^{2} P_{D} \Delta o_{D, n}^{2}
\end{aligned}
$$

11. Compute $\mathbb{E}\left\{\left|E_{D, n}\right|^{2}\right\}$ :

$\mathbb{E}\left\{\mathbf{q}_{D} \mathbf{q}_{D}^{H}\right\}=\alpha_{d}\left(1-\alpha_{d}\right) \operatorname{diag}\left(\mathbf{G}_{D} \mathbf{G}_{D}^{H}\right)\left(\operatorname{tr}\left(\mathbf{G}_{D} \mathbf{G}_{D}^{H}\right)\right)^{-1}$

$$
\begin{aligned}
\mathbb{E}\left\{\left|E_{D, n}\right|^{2}\right\} & =P_{D} \mathbb{E}\left\{\left|\Delta \mathbf{g}_{D, n}^{H} \mathbf{q}_{D}\right|^{2}\right\} \\
& =P_{D} \alpha_{d}\left(1-\alpha_{d}\right) \mathbb{E}\left\{\Delta \mathbf{g}_{D, n}^{H} \frac{\operatorname{diag}\left(\hat{\mathbf{G}}_{D} \hat{\mathbf{G}}_{D}^{H}\right)}{\operatorname{tr}\left(\hat{\mathbf{G}}_{D}^{H} \hat{\mathbf{G}}_{D}\right)} \Delta \mathbf{g}_{D, n}\right\} \\
& =P_{D} \alpha_{d}\left(1-\alpha_{d}\right) \mathbb{E}\left\{\Delta \mathbf{g}_{D, n}^{H} \operatorname{diag}\left(\frac{\hat{\mathbf{G}}_{D} \hat{\mathbf{G}}_{D}^{H}}{M}\right) \Delta \mathbf{g}_{D, n}\right\}\left(\operatorname{tr}\left(\frac{\hat{\mathbf{G}}_{D}^{H} \hat{\mathbf{G}}_{D}}{M}\right)\right)^{-1} \\
& =P_{D} \alpha_{d}\left(1-\alpha_{d}\right) \mathbb{E}\left\{\Delta \mathbf{g}_{D, n}^{H} \hat{\mathbf{D}}_{D} \Delta \mathbf{g}_{D, n}\right\}\left(\operatorname{tr}\left(\hat{\mathbf{D}}_{D}\right)\right)^{-1} \\
& =P_{D} \alpha_{d}\left(1-\alpha_{d}\right) \mathbb{E}\left\{\Delta \mathbf{g}_{D, n}^{H} \Delta \mathbf{g}_{D, n}\right\} \sum_{i=1}^{N} \beta_{D, i} \kappa_{D, i}\left(\operatorname{tr}\left(\hat{\mathbf{D}}_{D}\right)\right)^{-1} \\
& =P_{D} \alpha_{d}\left(1-\alpha_{d}\right) \Delta o_{D, n}^{2}
\end{aligned}
$$

12. Compute $\mathbb{E}\left\{\left|F_{D, n}\right|^{2}\right\}$ :

$$
\mathbb{E}\left\{\left|F_{D, n}\right|^{2}\right\}=P_{U} \sum_{i=1}^{N} \sigma_{i n}
$$

\section{Abbreviations}

ADC: Analog-digital converters; AGQN: Additive Gaussian quantization noise; AQNM: Additive quantization noise model; AWGN: Additive white Gaussian noise; BS: Base station; CSI: Channel state information; DAC: Digital-to-analog converters; FD: Full-duplex; IUI: Inter-user interference; LI: Loop interference; MIMO: Multi-input multi-output; MMSE: Minimum mean-square-error; MRC/MRT: Maximum ratio combining/maximum ratio transmission; RF: Radio frequency; SE: Spectral efficiency

\section{Funding}

The research was supported in part by the Postdoctoral Research Funding Plan in Jiangsu Province (grant no. 1501073B), Natural Science Foundation of Nanjing University of Posts and Telecommunications (grant no. NY218038), National Natural Science Foundation of China (61701254), Natural Science Foundation of Jiangsu Province (BK20170901), the Funds for International Cooperation and Exchange of the National Natural Science Foundation of China (61720106003), and the open research fund of National Mobile Communications Research, Southeast University (2017D06).

\section{Availability of data and materials}

We declared that materials described in the manuscript will be freely available to any scientist wishing to use them for non-commercial purposes, without breaching participant confidentiality.

\section{Authors' contributions}

$J \mathrm{~L}, J \mathrm{D}$, and JW designed the study, performed the research, analyzed the data, and wrote the paper. All authors read and approved the final manuscript.

\section{Competing interests}

The authors declare that they have no competing interests.

\section{Publisher's Note}

Springer Nature remains neutral with regard to jurisdictional claims in published maps and institutional affiliations.

\section{Author details}

${ }^{1}$ College of Telecommunications and Information Engineering, Nanjing University of Posts and Telecommunications, 210003 Nanjing, China. ${ }^{2}$ School of Science, Nanjing University of Posts and Telecommunications, Wenyuan Road, 210023 Nanjing, China. ${ }^{3}$ School of Engineering and Digital Arts, University of Kent, CT2 7NZ Canterbury, UK.

Received: 27 June 2018 Accepted: 30 August 2018

Published online: 15 September 2018

\section{References}

1. E. G. Larsson, O. Edfors, F. Tufvesson, T. L. Marzetta, Massive MIMO for next generation wireless systems. IEEE Commun. Mag. 52(2), 186-195 (2014)

2. J. Ma, S. Zhang, H. Li, N. Zhao, V. C. M. Leung, Interference-Alignment and Soft-Space-Reuse Based Cooperative Transmission for Multi-cell Massive MIMO Networks. IEEE Trans. Wirel. Commun. 17(3), 1907-1922 (2018)

3. Z. Zhang, K. Long, A. V. Vasilakos, Full-duplex wireless communications: challenges, solutions, and future research directions. Proc. IEEE. 104(7), 1369-1409 (2016)

4. H. Q. Ngo, E. G. Larsson, T. L. Marzetta, Energy and spectral efficiency of very large multiuser MIMO systems. IEEE Trans. Commun. 61(4), 1436-1449 (2013)

5. C. Pan, H. Zhu, N. J. Gomes, J. Wang, Joint precoding and RRH selection for user-centric green MIMO C-RAN. IEEE Trans. Wirel. Commun. 16(5), 2891-2906 (2017)

6. A. Mezghani, R. Ghiat, J. A. Nossek, in 2009 16th IEEE International Conference on Electronics, Circuits and Systems (ICECS 2009). Transmit processing with low resolution D/A-converters (IEEE, Yasmine Hammamet, 2009), pp. 683-686 
7. J. Singh, O. Dabeer, U. Madhow, On the limits of communication with low-precision analog-to-digital conversion at the receiver. IEEE Trans. Commun. 57(12), 3629-3639 (2009)

8. L. Fan, S. Jin, C.-K. Wen, H. Zhang, Uplink achievable rate for massive MIMO systems with low-resolution ADC. IEEE Commun. Lett. 19(12), 2186-2189 (2015)

9. J. Zhang, L. Dai, S. Sun, Z. Wang, On the spectral efficiency of massive MIMO systems with low-resolution ADCs. IEEE Commun. Lett. 20(5), 842-845 (2016)

10. X. D. Jia, M. Zhou, M. G. Xie, L. X. Yang, H. B. Zhu, Effect of low-resolution ADCs and loop interference on multi-user full-duplex massive MIMO amplify-and-forward relaying systems. IET Commun. 11(5), 687-695 (2017)

11. J. L. Jiao, X. D. Jia, M. Zhou, L. X. Yang, M. G. Xie, Full-duplex massive MIMO AF relaying system with low-resolution ADCs under ZFT/ZFR scheme. IEEE Adv. Inf. Technol. Electron. Autom. Control. Conf. (IAEAC). 2017, 2024-2028 (2017)

12. C. Pan, H. Mehrpouyan, Y. Liu, M. Elkashlan, A. Nallanathan, Joint pilot allocation robust transmission design for ultra-dense user-centric TDD C-RAN with imperfect CSI. IEEE Trans. Wirel. Commun. 17, 2038-2053 (2018)

13. C. L. Kong, C. J. Zhong, J. Shi, S. Yang, H. Lin, Multipair full-duplex massive MIMO relaying with low-resolution ADCs and imperfect CSI. IEEE Int Conf. Commun. (ICC). 2017, 1-6 (2017)

14. A. Mezghani, M. S. Khoufi, J. A. Nossek, in Proc. ITG/IEEE WSA. A Modified MMSE Receiver for Quantized MIMO Systems, (Vienna, 2007)

15. A. Mezghani, M. S. Khoufi, J. A. Nossek, in Proc. ITG/IEEE WSA. A Modified MMSE Receiver for Quantized MIMO Systems (Proc Itg/ieee Wsa, Vienna 2007)

16. X. M. Wang, D. M. Zhang, K. Xu, C. X. Yuan, On the Sum Rate of Multi-User Full-Duplex Massive MIMO Systems. IEEE Int. Conf. Commun. Syst. (ICCS). 2016, 1-7 (2016)

17. Q. Zhang, S. Jin, K. K. Wong, H. Zhu, M. Matthaiou, Power scaling of uplink massive MIMO systems with arbitrary-rank channel means. IEEE J. Sel. Topics Signal Process. 8(5), 966-981 (2014)

18. C. Pan, H. Zhu, N. J. Gomes, J. Wang, Joint user selection energy minimization for ultra-dense multi-channel C-RAN with incomplete CSI. IEEE J. Sel. Areas Commun, 1809-1824 (2017)

19. Y. Cao, N. Zhao, F. R. Yu, M. Jin, Y. Chen, J. Tang, V. C. M. Leung, Optimization or Alignment: Secure Primary Transmission Assisted by Secondary Networks. IEEE J. Sel. Areas Commun. 36(4), 905-917 (2018)

20. M. G. Sarret, G. Berardinelli, N. H. Mahmood, et al., Analyzing the potential of full duplex in $5 \mathrm{G}$ ultra-dense small cell networks. EURASIP J. Wirel. Commun Netw. 2016, 284-300 (2017)

21. J. Ma, S. Zhang, H. Li, N. Zhao, V. C. M. Leung, Base Station Selection for Massive MIMO Networks with Two-stage Precoding. IEEE Wirel. Commun. Lett. 6, 598-601 (2017)

\section{Submit your manuscript to a SpringerOpen ${ }^{\circ}$ journal and benefit from:}

- Convenient online submission

$\checkmark$ Rigorous peer review

- Open access: articles freely available online

- High visibility within the field

- Retaining the copyright to your article 